\title{
Prevalência dos Sintomas da Sindrome Pré-menstrual
}

\author{
Prevalence of symtoms in Premenstrual Syndrome \\ Clarissa Waldige Mendes Nogueira, João Luiz Pinto e Silva
}

\begin{abstract}
RESUMO
Objetivos: analisar os sintomas pré-menstruais relatados pelas mulheres com quadros graves de sindrome pré-menstrual (SPM), sua duração e época de aparecimento e patogênese.

Métodos: estudo de corte transversal por meio de questionário aplicado a 254 mulheres com idade entre 20 e 44 anos, sem doenças ginecológicas ou clinicas que comprometessem o estado geral ou bem-estar fisico. Foram excluidas as que estavam em amenorréia há mais de seis meses, as histerectomizadas e as grávidas.

Resultados: 110 mulheres (43,3\%) relataram pelo menos um sintoma intenso na fase prémenstrual causando danos à sua vida, sendo consideradas portadoras de SPM. A irritabilidade foi relatada por $86,4 \%$, cansaço por $70,9 \%$, depressão por $61,8 \%$, cefaléia por $61,8 \%$, mastalgia por 59,1\% e dor abdominal por 54,5\%. Quase todas (94,5\%) tinham mais de uma queixa, 89,1\% apresentaram sintomas psiquicos e mais de três quartos das pacientes (76,4\%) associavam queixas físicas e psíquicas. A maioria declarou duração de três a quatro dias $(32,4 \%)$ ou de cinco a sete dias $(31,4 \%)$.

Conclusões: o quadro clínico da SPM, apesar de multivariado, em geral é composto por irritabilidade e/ou depressão, associados a cansaço e dores de cabeça ou nas mamas, coexistindo sintomas físicos e psíquicos, por três a sete dias, sendo dificil atribuí-lo a uma etiologia única, à luz dos conhecimentos atuais.
\end{abstract}

PALAVRAS-CHAVE: Tensão pré-menstrual. Ciclo menstrual. Depressão.

\section{Introdução}

A sindrome pré-menstrual (SPM) é um complexo de sintomas que surge entre 10 e 14 dias antes da menstruação e desaparece com o início do fluxo, só se caracterizando como doença se afetar o dia-a-dia da mulher ${ }^{1}$. Já foram catalogados mais de 150 sintomas, que incidem de maneira variada e inconstante ${ }^{2}$.

Em relação à época de vigência, descrevemse dois padrões. Em um deles os sintomas aumentam gradualmente para desaparecerem com o início da menstruação e no outro a resolução é mais lenta, persistindo durante a fase folicular. Haveria ainda mais dois tipos, a incidência de sintomas graves apenas em alguns dias da fase

Departamento de Tocoginecologia da Faculdade de Ciências Médicas da Universidade Estadual de Campinas.

Correspondência:

Clarissa W. M. Nogueira

A/C - UNICAMP/ ASTEC/CAISM

Rua Alexander Fleming, 101 - Cidade Universitária "Zeferino Vaz"

13083-970 - Campinas - SP

Fone: (19) 788-9402 - Fax: (19) 289-5935 lútea ou em dois periodos distintos, por volta da ovulação e logo antes da menstruação ${ }^{3}$.

Segundo Bäckström ${ }^{4}$, a SPM se agrava com o início dos fenômenos regressivos do corpo lúteo, por volta do $24^{\circ}$ ou $25^{\circ}$ dia do ciclo. A surpreendente observação de Schmidt et al. ${ }^{5}$ de que os sintomas, tanto psíquicos quanto somáticos, continuaram a ocorrer na data projetada mesmo quando a menstruação foi induzida logo após a ovulação, por mifepristona ou luteólise por HCG, sugere que o evento desencadeador aconteça no primeiro período do ciclo.

Conforme a manifestação principal foram definidos quatro grupos: A, se predomina ansiedade, irritabilidade ou tensão nervosa; $\mathrm{H}$, se predominam edema, dores abdominais, mastalgia e ganho de peso; $\mathrm{C}$, com cefaléia, podendo ser acompanhada por aumento de apetite, desejo de doces, fadiga, palpitação e tremores, e finalmente o $\mathrm{D}$, se o quadro depressivo é preponderante, com insônia, choro fácil, esquecimento e confusão. Cada um estaria relacionado a fatores desencadeantes diferentes ${ }^{6}$.

Propomos analisar os sintomas e os dife- 
rentes quadros clínicos, sua duração e época de aparecimento, o que poderia contribuir para a discussão dos determinantes dessa síndrome.

\section{Pacientes e Métodos}

Foi realizado estudo de corte transversal com 254 mulheres de 20 a 44 anos que procuravam atendimento ginecológico de rotina ou eram acompanhantes de pacientes à consulta no Ambulatório do Hospital de Clínicas da Universidade Estadual de Campinas (UNICAMP). Elas foram abordadas sobre o motivo da consulta e aceitavam responder a um questionário sobre a presença de sintomas nas fases pré-menstrual e menstrual, sendo informadas dos objetivos da pesquisa e da desvinculação com o atendimento pretendido. Após concordância verbal buscava-se um local que permitisse manter a privacidade. Foram excluídas as mulheres com queixas ginecológicas, exceto corrimentos ou busca de método anticoncepcional, e também aquelas com problema de saúde que comprometesse o estado geral ou o bem-estar físico no momento da entrevista, amenorréia há mais de seis meses, história anterior de histerectomia e as grávidas.

Os dados foram apresentados em porcentagem. A SPM foi caracterizada como a ocorrência de sintomas intensos na fase pré-menstrual associados a danos causados por estes sintomas.

Por se tratar de método de entrevista, o procedimento ético baseou-se no consentimento verbal, após esclarecimento dos objetivos e da não-identificação da entrevistada. Os aspectos enfatizados foram a total desvinculação da aceitação com a consulta que procuravam e a possibilidade de utilização de um espaço resguardado para as perguntas de natureza íntima. Foram respeitados os direitos contemplados na Declaração de Helsinki (1990).

\section{Resultados}

Entre as entrevistadas, 43,3\% declararam algum sintoma intenso provocando prejuízos à sua vida diária (Tabela 1). A irritabilidade foi relatada por $86,4 \%$ delas, seguida por cansaço em $70,9 \%$ e depressão em $61,8 \%$. A cefaléia também foi assinalada por $61,8 \%$ das mulheres, mastalgia por $59,1 \%$ e dor abdominal por $54,5 \%$ (Tabela 2).
Tabela 1 - Distribuição das mulheres segundo freqüência e formas clínicas de síndrome prémenstrual (SPM).

\begin{tabular}{lcc}
\hline SPM & $\mathbf{n}$ & $\%$ \\
\hline Não & 35 & 13,8 \\
Leve & 57 & 22,4 \\
Moderada & 52 & 20,5 \\
Grave & 110 & 43,3 \\
Total & 254 & 100,0 \\
\hline
\end{tabular}

Tabela 2 - Número e percentual de mulheres com forma grave de SPM segundo os sintomas relatados.

\begin{tabular}{|c|c|c|}
\hline Sintoma & n (intensos) & $\%$ \\
\hline Irritação & $95 \quad(89)$ & 86,4 \\
\hline Cansaço & $78 \quad(45)$ & 70,9 \\
\hline Depressão & $68 \quad(44)$ & 61,8 \\
\hline Cefaléia & $68 \quad(40)$ & 61,8 \\
\hline Mastalgia & $65 \quad(34)$ & 59,1 \\
\hline Dor abdominal & $60 \quad(41)$ & 54,5 \\
\hline Dor nas costas & $40 \quad(23)$ & 36,4 \\
\hline Inchaço ou dor nas pernas & $28 \quad(21)$ & 25,4 \\
\hline Outros* & 69 & 62,7 \\
\hline
\end{tabular}

*Outros: indecisão (23), diminuição do desejo sexual (21), sintomas gastrointestinais (14), dores articulares ou generalizadas (5), insônia (3), batedeira, ansiedade ou palpitações (3).

Quase a totalidade $(94,5 \%)$ referiu mais de um sintoma. Apenas seis mulheres apresentavam sintoma único, três delas irritabilidade, duas cefaléia e uma cansaço. $26,3 \%$ associavam até quatro queixas e as restantes $68,2 \%$ tinham mais que isso. As queixas fisicas foram as únicas em $14(12,7 \%)$ entrevistadas e as psíquicas foram as únicas em 12 (10,9\%). Em 76,4\% houve associação dos dois tipos.

O quadro clínico mais comum foi composto de irritabilidade e/ou depressão associadas a cansaço e cefaléia ou dores na mamas, pernas, abdômen e costas (Tabela 3). Os sintomas eram restritos a um ou dois dias para $24,8 \%$. Outras $32,4 \%$ apresentavam sintomas por três a quatro dias. Mais $31,7 \%$ relataram sofrê-los por até sete dias e $11,4 \%$ por um período de sete a 14 dias (Tabela 4).

Tabela 3 - Número e percentual de mulheres com SPM conforme os quadros clínicos.

\begin{tabular}{lcc}
\hline Quadro Clínico & $\mathbf{n}$ & \% \\
\hline Com irritabilidade ou depressão intensa & 98 & 89,1 \\
Com dores intensas & 92 & 83,6 \\
Irritabilidade ou depressão e dores intensas & 78 & 70,9 \\
Irritabilidade ou depressão e cansaço & 73 & 66,4 \\
Irritabilidade ou depressão e cefaléia & 62 & 56,4 \\
\hline
\end{tabular}


Tabela 4 - Distribuição da duração dos sintomas nas mulheres com forma grave de SPM.

\begin{tabular}{lcc}
\hline Duração dos sintomas & $\mathbf{n}$ & $\%$ \\
\hline 1 ou 2 dias & 26 & 24,8 \\
3 a 4 dias & 34 & 32,4 \\
5 a 7 dias & 33 & 31,4 \\
8 a 14 dias & 12 & 11,4 \\
Total & 105 & 100,0 \\
\hline
\end{tabular}

Muitas mulheres com SPM (52,7\%) não souberam precisar a época de inicio de seus sintomas. As demais declararam apresentá-lo desde a menarca $(63,5 \%)$, após o casamento $(11,5 \%)$, após laqueadura $(9,6 \%)$ ou após a primeira gravidez $(9,6 \%)$ (Tabela 5$)$.

Tabela 5 - Distribuição da época em que se iniciou o quadro nas mulheres com SPM.

\begin{tabular}{lcc}
\hline Época de início & $\mathbf{n}$ & $\%$ \\
\hline Menarca & 33 & 63,5 \\
Após o casamento & 6 & 11,5 \\
Após a laqueadura & 5 & 9,6 \\
Após a primeira gravidez & 5 & 9,6 \\
Após uso de pílula & 3 & 5,8 \\
\hline
\end{tabular}

Obs.: Cinqüenta e oito mulheres (52,7\%) não souberam responder a essa questão.

\section{Discussão}

A presença de irritabilidade e/ou nervosismo, relatada por mais de $88 \%$ das mulheres com forma grave de SPM, constitui a face mais característica do quadro. Enquanto um estudo sueco detectou ocorrência de irritabilidade pré-menstrual moderada em $31 \%$ e grave em 3\% das mulheres ${ }^{7}$, quase $40 \%$ de nossas entrevistadas indicaram esse sintoma intensamente, o que nos remete a diferenças culturais que poderiam influir no desenvolvimento do problema.

$\mathrm{Na}$ seqüência, os sintomas mais relacionados foram cansaço, em 70,9\% dos casos, e depressão em $61,8 \%$. A fadiga tem sido atribuída à hipoglicemia, alteração no equilíbrio das prostaglandinas ou a disfunção tiroidiana ${ }^{8,9}$, ao passo que a depressão tem sido relacionada à deficiência de vitamina B6 ou, mais especificamente, de serotonina ${ }^{10,11}$.

Apenas três mulheres que relataram depressão não se queixavam também de irritabilidade e pouco mais de $10 \%$ de todo o grupo não apresentavam um desses sintomas, que poderiam ser facetas do mesmo quadro, pois existem depressões sem distúrbio de humor, ditas "mas- caradas", o que poderia ser o processo subjacente nas pacientes irritadiças.

Foi sugerido que um estado disfórico experimentado repetidamente poderia facilitar o desenvolvimento gradual ou a expressão de uma desordem afetiva, em indivíduos geneticamente predispostos $^{12}$. Portanto, a depressão pré-menstrual tanto poderia preceder o desenvolvimento de depressão maior, como ser manifestação de resolução incompleta dessa patologia, sendo o período apenas de maior vulnerabilidade biológica $^{13}$.

A cefaléia foi assinalada por $61,8 \%$ das mulheres com forma grave de SPM, dado semelhante ao constatado em um estudo prospecti$v^{14}{ }^{14}$ e em uma avaliação retrospectiva ${ }^{15}$ na qual se observou essa queixa em $86 \%$ das mulheres.

A cefaléia poderia ser manifestação da enxaqueca menstrual, que incide na semana anterior ou especialmente nos primeiros três dias da menstruação ${ }^{16}$, correspondendo a $8 \%$ das enxaquecas. É atribuída a distúrbio da transmissão serotoninérgica, sendo observada queda nos níveis de $5-\mathrm{OH}$-triptamina nas plaquetas no início do quadro ${ }^{17}$. O estrogênio estimularia a produção de adrenoceptor na vasculatura, bloquearia a recaptação da serotonina, inibindo a enzima da degradação da monoaminoxidase, levando a vasodilatação das artérias cerebrais, por liberação de peptídeos vasoativos ${ }^{18}$.

Um sintoma muito incômodo e igualmente bastante relatado é a mastalgia, que além do inconveniente da dor, causa prejuízos ao relacionamento amoroso e temor de sua ligação com neoplasia. Embora tradicionalmente não seja considerada indicadora de perigo, há relatos de maiores indices de sensibilidade pré-menstrual em doentes com câncer de mama, após controlado o efeito de outros fatores ${ }^{19}$.

A mastalgia tem sido atribuída a niveis alterados de prolactina, que alguns advogam ser a causa da SPM, por ter sido constatada elevação em mais de $40 \%$ das doentes ${ }^{20}$. Sua secreção parece estar sob dependência frenadora do neurotransmissor dopamina e a hiperprolactinemia indicaria redução da sua atividade, que é relacionada por sua vez ao desenvolvimento de depressão ${ }^{21}$. Recentemente tem sido invocado um desequilíbrio nas prostaglandinas e recomendada a administração de ácidos graxos essenciais para correção ${ }^{22}$.

A dor abdominal, apontada por $54,5 \%$ dessas mulheres, poderia derivar de dificuldade das pacientes em discriminar esse quadro da dismenorréia ou ser manifestação de processo congestivo pélvico, constipação intestinal ou distúrbio autonômico, relacionados ao aumento da 
progesterona, à ação de prostaglandinas ou vasopressina, que também poderiam ter papel nas dores lombares, presentes em $36,4 \%$ do grupo afetado.

O inchaço nas pernas foi registrado por $34,5 \%$ do grupo com SPM e a dor nas pernas aludida espontaneamente por mais $13,6 \%$. Os indices de edema pré-menstrual relatados na literatura chegam a $92 \%$, sendo atribuído à retenção hídrica, por ação da progesterona provocando flacidez da parede venosa, com prejuízo à drenagem e também por atuar como agonista da aldosterona induzindo natriurese, imediatamente compensada por aumento de renina ou conversão para desoxicorticosterona ${ }^{23}$. Recentemente apontaram-se evidências de haver redistribuição de líquidos e não retenção ${ }^{24}$.

A distinção entre as esferas somática e psíquica não é fácil, mas nesta avaliação mais de $75 \%$ apresentavam sintomas dessas duas naturezas, associando irritabilidade ou depressão com quadros dolorosos ou edema. A maioria das entrevistadas não soube precisar quando haviam iniciado seus sintomas, levando a pensar em processo insidioso, que demora a ser percebido. Entre as que se lembravam, $63,5 \%$ os percebiam desde as primeiras menstruações e as restantes após o casamento, primeira gravidez, laqueadura ou uso da pílula anticoncepcional. Esses dados também são encontrados na literatura, diferindo o papel da primeira gravidez, geralmente mais intenso. ${ }^{25}$

Um terço das mulheres com SPM revelou sofrimento de apenas um ou dois dias e mais de $10 \%$ declararam vigência por mais de uma semana. As $63,8 \%$ restantes foram afetadas por três a sete dias. A multiplicidade de sintomas, experimentada por mais de 94\%, não necessariamente concomitantes, poderia interferir na duração do quadro.

As inúmeras teorias etiológicas para a SPM são alicerçadas em ensaios clínicos ou experimentais, mas nenhuma consegue explicar tão múltiplos sintomas e formas de apresentação. Não foi possivel configurar quadros bem delimitados, que pudessem indicar etiologia comum. É possivel que seja a manifestação ou agravamento de doença preexistente, tendo a segunda fase do ciclo maior vulnerabilidade biológica.

O quadro clínico da SPM em geral compõese de irritabilidade e/ou depressão, que podem ser acompanhados de cansaço e dores de cabeça ou nas mamas, quase sempre havendo associação de sintomas físicos e psíquicos, com duração de três a sete dias, sendo difícil atribuí-lo a uma etiologia única, à luz dos conhecimentos atuais.

\section{SUMMARY}

Purpose: to analyze the symptoms of premenstrual syndrome (PMS), its duration, time of presentation and pathogenesis.

Methods: a questionnaire was applied to 254 women aged 20 to 44 years, without gynecological or clinical conditions affecting their general state or physical well-being, excluding those with amenorreha for more than six months, hysterectomized or pregnant.

Results: one hundred and ten women (43.3\%) describing at least one intense symptom causing harm to their life, were considered as having PMS. Irritability was presented by $86.4 \%$, tiredness by $70.9 \%$, depression by $61.8 \%$, headache by $61.8 \%$, breast tenderness by $59.1 \%$ and abdominal pain by 54.5\%. Almost all (94.5\%) had more that one symptom, $89.1 \%$ presented psychological complaints, and $76.4 \%$ associated physical and psychological complaints. Most declared that duration of the symptoms was three to four days (32.4\%) or five to seven days $(31.4 \%)$.

Conclusions: the clinical picture of PMS is generally composed of irritability and/or depression, associated with tiredness and headache or breast pain, there being association of physical and psychological symptoms, for 3 to 7 days, and it is difficult to attribute a single etiology to it at this stage of knowledge.

KEY WORDS: Premenstrual syndrome. Premenstrual tension. Menstrual cycle. Depression.

\section{Referências}

1. Dalton K. The premenstrual syndrome and progesterone therapy. $2^{\text {nd }}$ ed. Chicago: Year Book Medical Publishers; 1984. p.169.

2. Budoff PW. Use of prostaglandin inhibitors in the treatment of PMS. Clin Obstet Gynecol 1987; 30:453-65.

3. Mortola JF. Issues in the diagnosis and research of premenstrual syndrome. Clin Obstet Gynecol 1992; 35:587-98.

4. Bäckström T. Neuroendocrinology of premenstrual syndrome. Clin Obstet Gynecol 1992; 35:612-28

5. Schmidt PJ, Nieman LK, Grover GN, Muller KL, Merrian GR, Rubinow DR. Lack of effect of induced menses on symptoms in women with premenstrual syndrome. N Engl J Med 1991; 324:1174-9.

6. Hargrove JT, Abraham GE. The incidence of premenstrual tension in a gynecologic clinic. J Reprod Med 1982; 27:721-4. 
7. Andersch B, Wendestam C, Hahn L, Oehman R. Premenstrual complaints. I. Prevalence of premenstrual symptoms in a Swedish urban population. J Psychosom Obstet Gynaecol 1986; 5:39-49.

8. Reid RL, Yen SS. Premenstrual syndrome. Am J Obstet Gynecol 1981; 139:85-104.

9. Hall RC. Psychiatric effects of thyroid hormone disturbance. Psychosomatics 1983; 24:7-18.

10.Rapkin AJ. Neuroanatomy, neurophysiology and neuropharmacology of pelvic pain. Clin Obstet Gynecol 1990; 33:119-29.

11.Shaarawy M, Shaaban HA, Eid MM, Abdel-Aziz O. Plasma beta-endorphin level in cases of luteal phase defect. Fertil Steril 1991; 56:248-53.

12.Rubinow DR, Schmidt PJ. The treatment of premenstrual syndrome: forward into the past. N Engl J Med 1995; 332:1574-5.

13.Pearlstein TB. Hormones and depression: what are the facts about premenstrual syndrome, menopause and hormone replacement therapy? Am J Obstet Gynecol 1995; 173:646-53.

14.Hagen I, Nesheim BI, Tuntland T. No effect of vitamin B6 against premenstrual tension. A controlled clinical study. Acta Obstet Gynecol Scand 1985; 64:667-70.

15.Bedone AJ, Cappi-Maia EM, Monteiro IMU. Tensão pré-menstrual: resultados clínicos com cinco tipos de tratamento. Rev Bras Ginecol Obstet 1995; 17:295-303.

16.Johannes CB, Linet MS, Stewart WF, Celentano DD, Lipton RB, Szklo M. Relationship of headache with the phase of the menstrual cycle among young women: a daily diary study. Neurology 1995; 45:1076-82.

17.Raskin NH. Serotonin receptors and headache. N Engl J Med 1991; 325:353-4.

18.Digre K, Damasio H. Menstrual migraine: differential diagnosis, evaluation and treatment. Clin Obstet Gynecol 1987; 30:417-30.

19.Goodwin PJ, DeBoer G, Clark RM, et al. Cyclical mastopathy and premenopausal breast cancer risk. Results of a case-control study. Breast Cancer Res Treat 1995; 33:63-73.

20.Halbreich U, Endicott J, Lesser J. The clinical diagnosis and classification of premenstrual changes. Can J Psychiatry 1985; 30:489-97.

21.Alila HW, Rogo KO, Gombe S. Effects of prolactin on steroidogenesis by human luteal in cell culture. Fertil Steril 1987; 47:947-55.

22.Horrobin DF. The role of essential fatty acids and prostaglandins in the premenstrual syndrome. J Reprod Med 1983; 28:465-8.

23.Augusto N, Urbanetz AA. Síndrome de tensão prémenstrual. I. Aspectos clínicos e fisiopatologia. Femina 1985; 13:682-90.

24.Tollan A, Oian P, Fadnes HO, Maltau JN. Evidence for altered transcapillary fluid balance in women with the premenstrual syndrome. Acta Obstet Gynecol Scand 1993; 72:238-42.

25.Keye WR Jr, Hammond DC, Strong T. Medical and psychologic characteristics of women presenting with premenstrual symptoms. Obstet Gynecol 1986; 68:634-7.

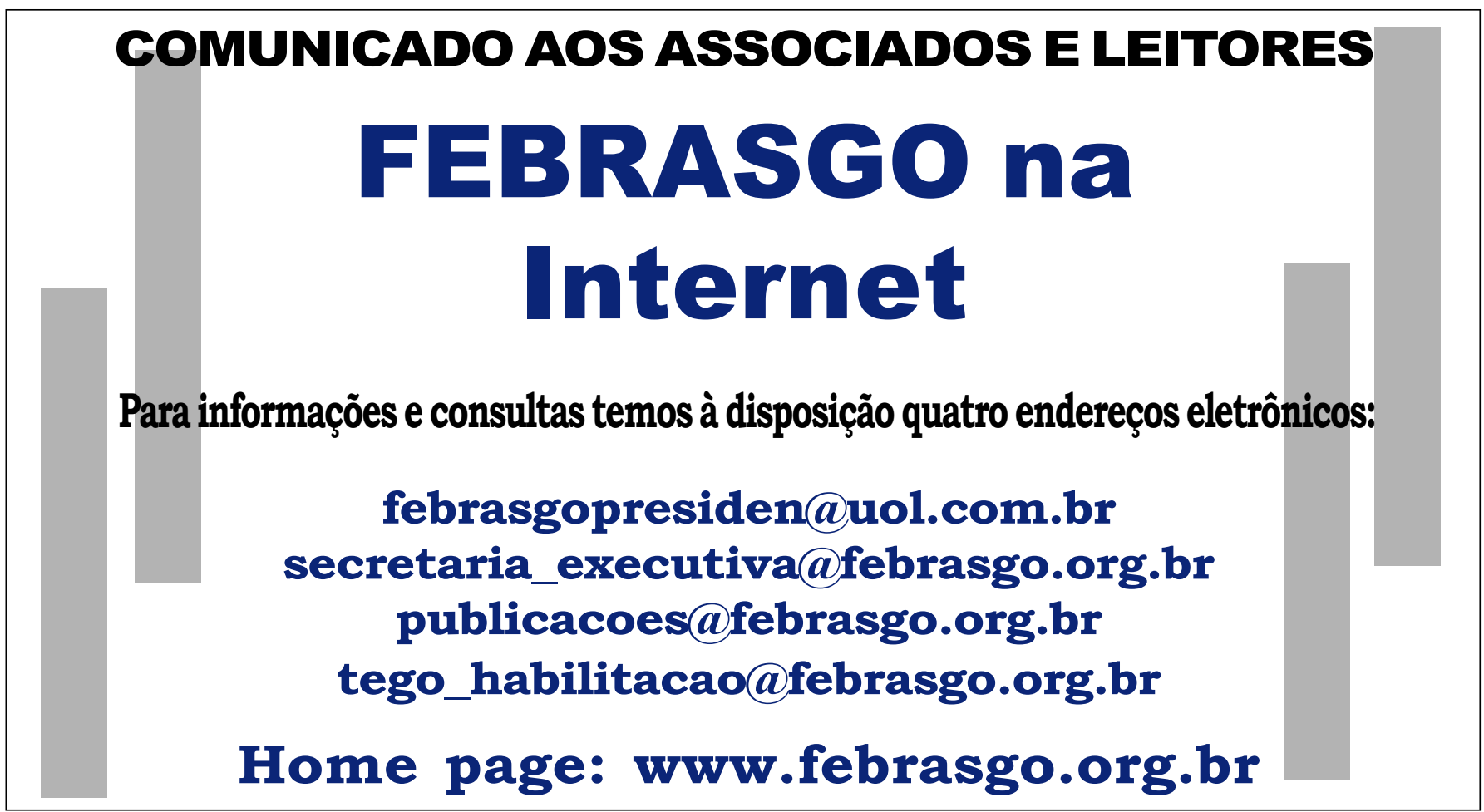

